\title{
Probing the Interiors of Very Hot Jupiters Using Transit Light Curves
}

\author{
Aaron S. Wolf and Darin Ragozzine \\ Division of Geological and Planetary Sciences, California Institute of Technology, Pasadena, \\ CA 91125 \\ email: awolf@gps.caltech.edu and darin@gps.caltech.edu
}

\begin{abstract}
Accurately understanding the interior structure of extra-solar planets is critical for inferring their formation and evolution and resolving the origin of anomalous planetary radii. The internal density distribution of the planet has a direct effect on the star-planet orbit through the gravitational quadrupole of rotational and tidal bulges, measured by the planetary Love number $\left(k_{2 p}\right.$, twice the apsidal motion constant). We find that the quadrupole of the planetary tidal bulges dominates the rate of apsidal precession of single very hot Jupiters by more than an order of magnitude over general relativity and the stellar quadrupole. For the shortest-period planets, the planetary interior induces precession of a few degrees per year. By investigating the full photometric signal of apsidal precession, we find that transit timing induces a relatively small signal compared to the changes in transit shapes. With its long baseline of ultra-precise photometry, the future space-based Kepler mission should be able to realistically detect the presence or absence of a core in very hot Jupiters with orbital eccentricities as low as $e \sim 0.001$. We show that the signal due to $k_{2 p}$ is not degenerate with other parameters and has a unique signature on the transit light curve. This technique, outlined in more detail in Ragozzine \& Wolf 2008 provides the first readily employed method for directly probing the interiors of extra-solar planets.
\end{abstract}

\section{Introduction}

As seen from several contributions to this volume, there is great interest in understanding the interiors of extra-solar planets. Transiting planets, with direct measurements of both mass and radius, are especially useful in this regard.

The inferred interior structures of transiting planets are generally in accord with extrapolations of models of our own giant planets. However, it has become clear that some planets do not follow these trends and require additional fine-tuning of various model parameters (Guillot et al. 2007 and Burrows et al. 2007). Nevertheless, the current goal is to use transiting planets as an ensemble to minimize model-based assumptions and thus improve our understanding of their bulk composition, internal dynamics, and formation histories.

Our progress in this endeavor would greatly benefit from model-independent measures of interior structure. The idea of obtaining direct structural measurements for distant objects is by no means a new one. For decades, the interiors of eclipsing binary stars have been measured by observing apsidal motion, i.e. precession of the orbit due to the non-point-mass component of the gravitational field (Sterne 1939). The signal of the changing orbit is encoded in the light curves of these systems.

We have applied these techniques to extra-solar transiting planets and find that probing the interiors is quite plausible with high-precision long-baseline photometry. In particular, we focus our analysis on the data expected from NASA's Kepler mission to be launched in early 2009 (Borucki et al. 2008). Kepler will obtain exquisite photometry on $\sim 100,000$ 
stars, of which about 30 are expected to host very hot Jupiters (Beatty \& Gaudi 2008). We find that Kepler has the potential to measure the gravitational quadrupoles of very hot Jupiters. If successful, this will constitute a major step towards an understanding of the diversity of planetary interiors.

Here we summarize the results of Ragozzine \& Wolf (2008), in preparation (RW08). RW08 describe the background theory, transit light curve model, and other relevant topics in much greater detail.

\section{Effect of Interiors on the Planetary Orbit}

The internal structure of very hot Jupiters has a direct effect on their orbit. This allows us to probe the internal density distribution by observing small variations in the orbit as evidenced by subtle changes in transit photometry. The primary effect of structure is to induce a linear drift in the angle describing the periapse position for eccentric orbits, $\omega$. This apsidal precession causes the orientation of the planetary orbit to change with respect to Earth, modifying the transit light curve as described in Section 3 below.

The strength of the planetary quadrupole is determined by the size of the rotational bulge and the time-varying tidal bulge. Sterne (1939) showed that the effect of these bulges on apsidal precession can be described by the planetary Love number $k_{2 p}$ which is twice the "apsidal motion constant" (also called $k_{2}$ ). There is a direct relation between $k_{2}$ and $J_{2}$; they are effectively interchangeable (RW08). This probe into interior structure has been used in eclipsing binary stars for decades and was one of the first indications that stars were highly centrally condensed, with $k_{2} * \simeq 0.03$ typical for main sequence solar-like stars (Claret 1995). This is contrasted with the maximum $k_{2}$ of $3 / 2$ for an object with uniform density.

Planets have much higher values of $k_{2}$ than stars since they are not as centrally condensed. For example, Jupiter has $k_{2 J} \simeq 0.49$. Saturn, which has a much larger core (relative to its mass) of $k_{2 S} \simeq 0.32$. From this, we can see that planets with and without significant cores differ in $k_{2 p}$ by about $\sim 0.1$. This can also be inferred from Bodenheimer et al. (2001) and Barnes \& Fortney (2003) by using the Darwin-Radau relation to approximately convert the given moment of inertia factor to $k_{2}$. For plausible interior models, $k_{2 p}$ can range quite widely, from 0.1 to 0.6 , implying that even an imprecise measurement of $k_{2 p}$ will be a valuable constraint on interior models of extra-solar planets.

With this in mind, we can now calculate the expected apsidal precession of the orbits of extra-solar planets. The main contributors are the rotational and tidal potentials of the planet and star, along with general relativity. To first-order, these sources of apsidal precession simply add to give the total precession rate. In order of importance for very hot Jupiters and as given by Sterne (1939):

$$
\begin{aligned}
\dot{\omega}_{\mathrm{tot}} & =\dot{\omega}_{\mathrm{tid}, \mathrm{p}}+\dot{\omega}_{\mathrm{GR}}+\dot{\omega}_{\mathrm{rot}, \mathrm{p}}+\dot{\omega}_{\mathrm{rot}, *}+\dot{\omega}_{\mathrm{tid}, *} \\
& =\frac{15}{2} k_{2 p}\left(\frac{R_{p}}{a}\right)^{5} \frac{M_{*}}{M_{p}} f_{2}(e) n \\
& +\frac{3 G M_{*} n}{a c^{2}\left(1-e^{2}\right)} \\
& +\frac{k_{2 p}}{2}\left(\frac{R_{p}}{a}\right)^{5} \frac{\nu_{p}^{2} a^{3}}{G M_{p}} g_{2}(e) n
\end{aligned}
$$




$$
\begin{aligned}
& +\frac{k_{2 *}}{2}\left(\frac{R_{*}}{a}\right)^{5} \frac{\nu_{*}^{2} a^{3}}{G M_{*}} g_{2}(e) n \\
& +\frac{15}{2} k_{2 *}\left(\frac{R_{*}}{a}\right)^{5} \frac{M_{p}}{M_{*}} f_{2}(e) n
\end{aligned}
$$

where subscripts $p$ and $*$ refer to the planet and star, respectively, $R$ is the radius, $M$ is the mass, $a$ is the semi-major axis, $n$ is the mean motion (i.e. $\left.n=\frac{2 \pi}{P_{\text {orb }}}\right), c$ is the speed of light, $e$ is the eccentricity, $\nu$ is the spin angular frequency (i.e. $\nu=\frac{2 \pi}{P_{\mathrm{spin}}}$ ), and $f_{2}(e)$ and $g_{2}(e)$ are functions:

$$
\begin{gathered}
f_{2}(e)=\left(1-e^{2}\right)^{-5}\left(1+\frac{3}{2} e^{2}+\frac{1}{8} e^{4}\right) \approx 1+\frac{13}{2} e^{2}+\frac{181}{8} e^{4}+\ldots \\
g_{2}(e)=\left(1-e^{2}\right)^{-2} \approx 1+2 e^{2}+3 e^{4}+4 e^{6}+\ldots
\end{gathered}
$$

Taking measured or estimated parameters from the known transiting planets and assuming psuedosynchronous rotation, Figure 1 shows the fraction of total apsidal precession due solely to the planet. We find that for very hot Jupiters, the planetaryinduced precession strongly dominates over other contributions by more than an order of

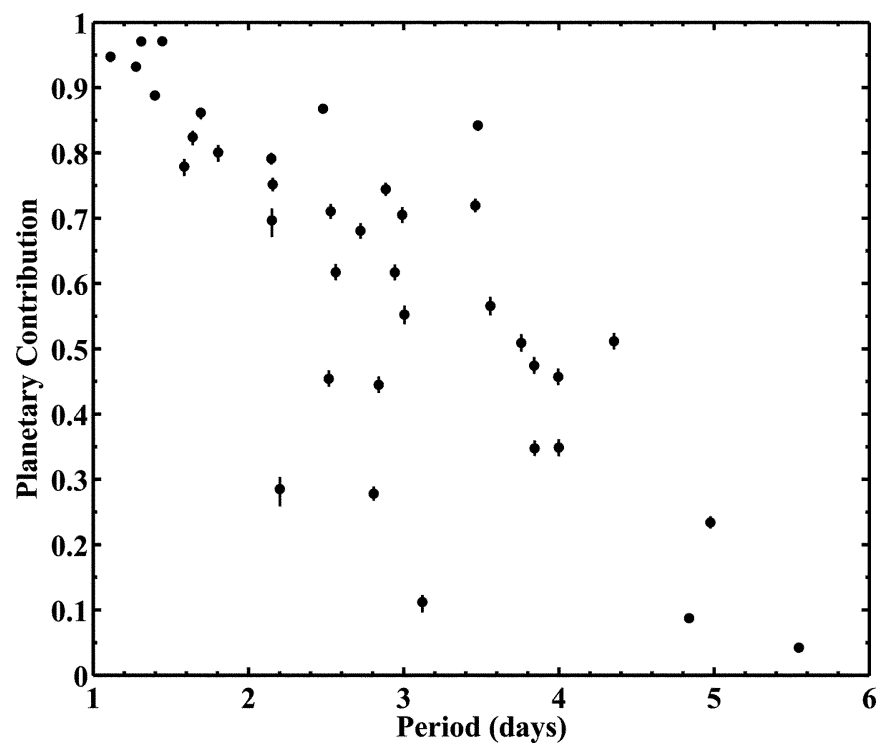

Figure 1. Fraction of Apsidal Precession Due to the Planetary Quadrupole. The points show the planetary fraction of the total apsidal precession (Eq. 2.1) calculated for the known transiting extra-solar planets with properties taken from F. Pont's website (http://www.inscience.ch/transits/simpleTABLE.dat) and J. Schneider's Exoplanet Cata$\log$ (http://www.exoplanet.eu), assuming the planet has a typical Love number of $k_{2 p}=0.3$. The apsidal precession induced by the tidal and rotational bulges of the planet overcome precession due to general relativity and the star, especially for short period planets. The "error bars" show the range of planetary contributions for a $5 \%$ variation in stellar masses (and hence $\dot{\omega}_{\mathrm{GR}}$ ) and the comparatively smaller effect of varying the stellar Love number and rotation rate over all reasonable values. The four planets where the planetary contribution to apsidal precession is most important also have the shortest precession periods: CoRoT-Exo-1b, WASP-4 b, TrES-3 b, and OGLE-TR-56 b would fully precess in about 93, 81, 184, and 87 years, respectively. In all reasonable cases, the dominant signal in apsidal precession of very hot Jupiters is $k_{2 p}$, which is determined by the internal density distribution and is a powerful probe into the interior structure. 
magnitude. This is mostly due to the very strong tidal bulge raised by the nearby star which, in the best cases, induces precession of a few degrees per year. The precession due to the planet has generally been neglected in extra-solar planet transit timing work to date (Miralda-Escudé 2002 and Heyl \& Gladman 2007), which has considered stellar oblateness or general relativity to be the dominant effect (in the absence of other planets) - though Jordan \& Bakos (2008) have independently pointed out that $\dot{\omega}_{\text {tidal,p }}$ can be an important source of apsidal precession. Hence, measuring apsidal precession of very hot Jupiters essentially gives $\dot{\omega}_{\text {tidal,p }}$ which is directly proportional to $k_{2 p}$, implying that transit light curve variations due to apsidal precession can directly probe the interiors of extra-solar planets.

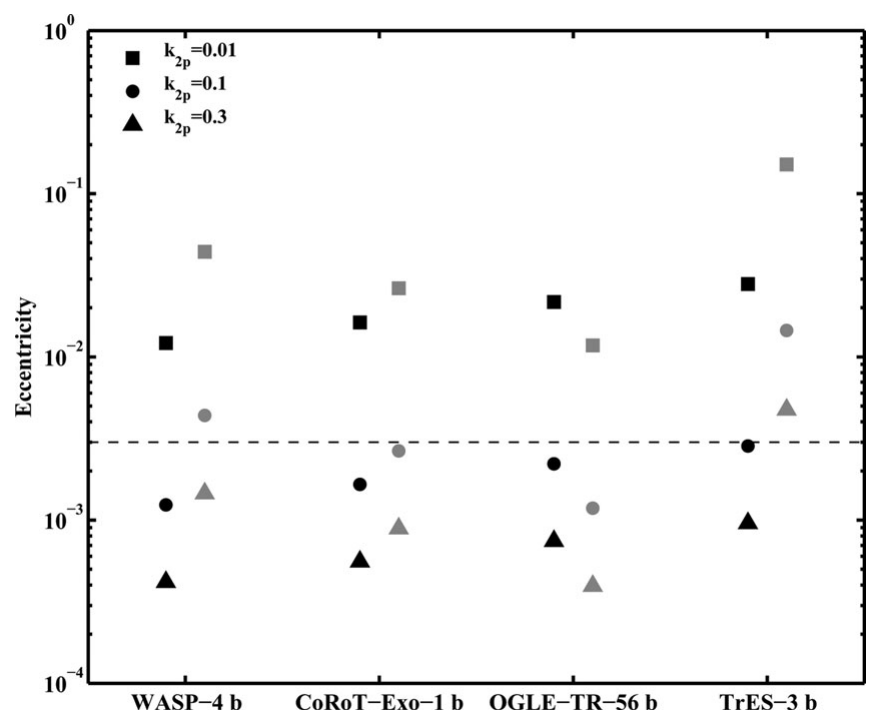

Figure 2. Eccentricities Needed to Detect Interior Properties from Apsidal Precession. The best-known planets for detecting $k_{2 p}$ precession are the very hot Jupiters WASP-4 b, CoRoT-Exo-1 b, OGLE-TR-56 b, and TrES-3 b. For each of these planets (using the parameters in Table 1), we determined the threshold $k_{2 p}$ value for a variety of eccentricities. Apsidal precession is much easier to detect for larger eccentricities, so that increasing $e$ decreases the detectable $k_{2 p}$. (See discussion in text.) Black symbols correspond to calculations with $\omega=0^{\circ}$ and gray symbols correspond to $\omega=90^{\circ}$. Interpolating on our results, the graph identifies the eccentricities required to detect precession due to a "typical" planetary interior of $k_{2 p}=0.3$ (triangles). (For example, when $e=0.0004$, the apsidal precession due to the planet should be just detectable.) A higher eccentricity would be needed to measure $k_{2 p}$ with sufficient accuracy to distinguish between a massive core and a core-less model (circles). Systematic errors are expected to become important once the measurement error on $k_{2 p}$ reaches as low as $\sim 0.01$ (squares). The dotted line at $e=0.003$, illustrates the very low eccentricities that are plausible for these objects as estimated from the measurement of $e \cos \omega=0.001 \pm 0.0002$ for very hot Jupiter HD 189733b (Knutson et al. 2007 and see also Agol et al. in this volume). If any of the $\sim 30$ very hot Jupiters (Beatty \& Gaudi 2008) to be discovered by Kepler have such an eccentricity, the long-term high-precision photometry would allow a powerful probe into planetary interiors.

\section{Transit Light Curves of Apsidal Precession}

Given the strength of apsidal precession due to the planet and the scientific value of determining $k_{2 p}$, we chose to analyze the observability of apsidal precession in very hot Jupiters by investigating full simulated light curves. In particular, we considered 


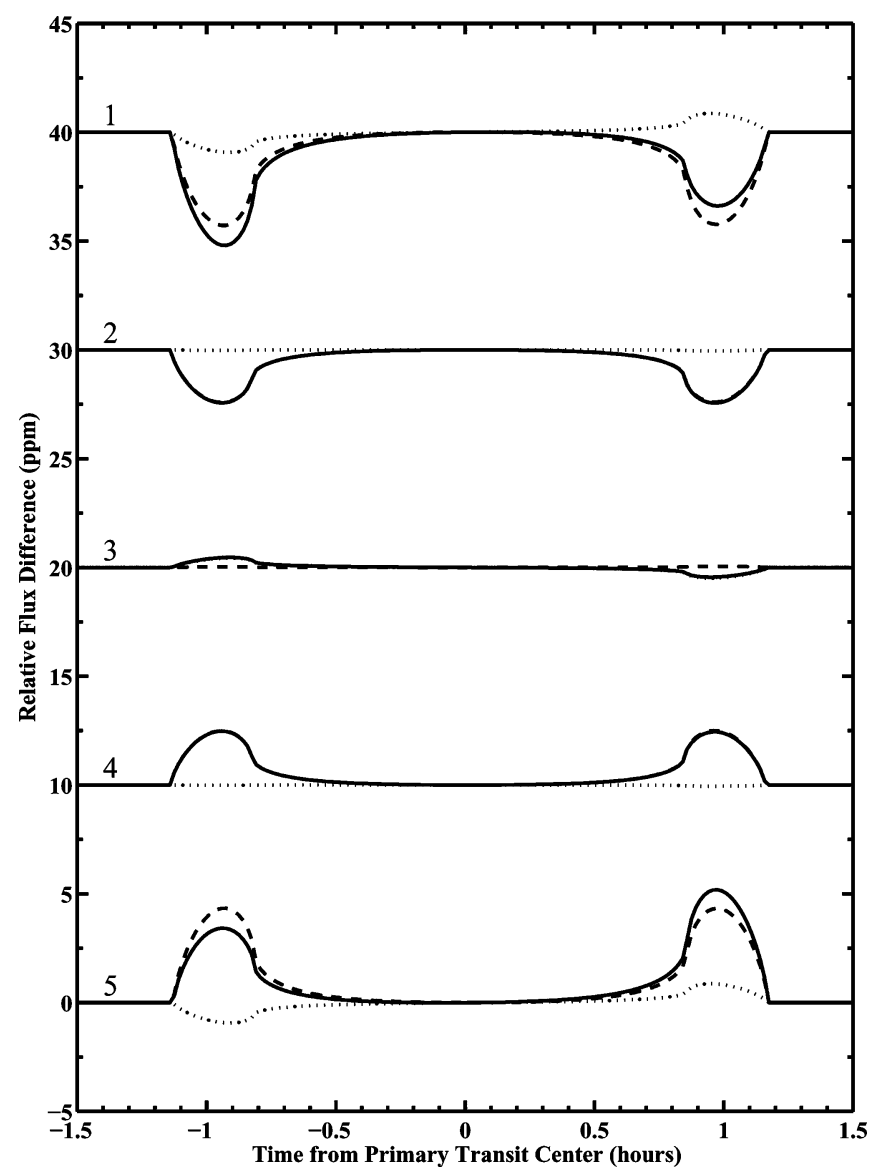

Figure 3. Excerpts of Photometric Difference Signal. As described in the text, to measure the observability of $k_{2 p}$, we subtract a model transit light curve with non-zero $k_{2 p}$ from the light curve with $k_{2 p}=0$. Here we show the difference signal for WASP-4b with an eccentricity of $0.001, \omega=0^{\circ}$, and $k_{2 p}=0.122$. At this value of $k_{2 p}$, the total effective signal-to-noise is 1 on a moderately bright star $(V=12)$. The effects of transit timing and transit "shaping" can both be seen. Transit timing has an asymmetric signal (dotted lines), obtained when subtracting two transit curves slightly offset in time. Transit shaping, which is due to changing transit duration, creates a symmetric signal (dashed lines). The total difference signal (solid lines) is totally dominated by the effect of transit shaping, which has $\sim 30$ times more signal than transit timing alone. Both effects are maximized at the beginning (1) and end (5), as expected for a signal that increases with longer baseline. The maximal signal occurs during ingress and egress, when the light curve changes the fastest. The transit shapes are equivalent at the center (3) by construction. The transit timing anomaly of precession is quadratic, which, when fitted with a best-fit straight line corresponding to a non-precessing signal, yields two intersections when transit timing is minimized $(2,4)$. The transit timing offset at the beginning and end is only 0.037 seconds, while the center is offset by -0.018 seconds. See RW08 for more details.

a simulated Kepler light-curve of observations with a 1-minute cadence for 3.5 years. The details of our photometric model are described in RW08 and include accounting for the difference between mean and osculating elements (Kozai 1959) as well as secondary transits in the optical (López-Morales \& Seager 2007).

Using this model, we calculate full Kepler photometry for known planets; these are not in the Kepler field, but should be considered as analogs of the $\sim 30$ very hot Jupiters to be discovered (Beatty \& Gaudi 2008). In particular, we subtract a base model with 
$k_{2 p}=0$ from a model with non-zero $k_{2 p}$. By taking the root sum of squares of the residuals (Figure 3) and comparing it to the photometric accuracy of Kepler (400 parts per million per 1 minute cadence on a $V=12$ star), we are able to find the "threshold" $k_{2 p}$ that yields an effective signal-to-noise of 1 . We modify all the parameters (except the eccentricity) to minimize or eliminate all residual signal not due to $k_{2 p}$. See RW08 for more details. We repeat this process for different values of eccentricity and at two different orientations: $\omega=0^{\circ}$ and $\omega=90^{\circ}$.

At $\omega=0^{\circ}$, the dominant source of signal is "transit shaping" (Figure 3), secular changes in the shapes of transits from changing transit duration and orientation (MiraldaEscudé 2002, Jordan \& Bakos 2008, and Pál \& Kocsis 2008). This signal is 30 times stronger than transit timing, which has been the focus of many theoretical and observational works to date. For analogs of currently known very hot Jupiters, we find that $k_{2 p}$ can be measured with enough accuracy to ascertain the presence of a massive core as long as the eccentricity is as high as $\sim 0.001$ (Figure 2), similar to the measured eccentricity of HD189733b.

At $\omega=90^{\circ}$, the dominant source of signal is the changing offset between primary and secondary transit times. Secondary transits for very hot Jupiters can be relatively deep ( $400 \mathrm{ppm})$, even in Kepler's optical bandpass. Unless the secondary transit is particularly deep (OGLE-TR-56 b, 1100 ppm), in this orientation larger eccentricities are required to accurately measure $k_{2 p}$ (Figure 2, gray symbols).

\section{Interpretation of $k_{2 p}$ signal}

The value of $k_{2 p}$ is directly invertible from $\dot{\omega}_{\text {tot }}$ with little error since all the other parameters in Equation 2.1 are either small or well-known from photometry and reasonable radial velocity measurements. In addition, the signal due to this rate of apsidal precession is unique and cannot be confused with additional planets (RW08). With very low planetary obliquities expected from tidal damping, we have also verified that nodal precession is a relatively small signal.

Tidal semi-major axis decay causes similar transit time deviations, but without the associated transit shaping: hence, it is a qualitatively different signal. However, if the estimate of $\dot{P} \approx 1 \mathrm{~ms} / \mathrm{yr}$ from Sasselov (2003) is correct, semi-major axis decay can be a significant source of non-Keplerian signal. Using our model, we estimate that for very hot Jupiters, a change in period of $\dot{P} \simeq 0.5 \mathrm{~ms} /$ yr is detectable with Kepler (RW08). This signal is present at any value of eccentricity and includes removing degeneracies due to other parameters as in the calculation of threshold $k_{2 p}$. This result is quite exciting as Kepler should be able to measure or place strong constraints on $Q_{*}$ for the first time for a variety of stars.

\section{Conclusions}

As detailed in RW08, with a high enough eccentricity ( $\gtrsim 0.001)$, the planetary Love number of very hot Jupiters has an observationally detectable signature and can provide a new and unique probe into the interiors of very hot Jupiters. The internal density distribution of the planet, characterized by $k_{2 p}$, has a large and clear signal, distinct from any other parameters or phenomena.

In analyzing the photometric signal of apsidal precession, we find that transit timing variations are an almost negligible source of signal. The effect of "transit shaping" has $\sim 30$ times the photometric signal of transit timing (Figure 3) for apsidal precession (see also Jordan \& Bakos 2008 and Pál \& Kocsis 2008). At orientations where transit timing 
and shaping are weakest, the changing offset between primary and secondary transit times can be used to measure $k_{2 p}$.

Accurately measuring the interior structure of distant extra-solar planets seems too good to be true. Nevertheless, the exquisite precision, constant monitoring, and 3.5-year baseline of the Kepler mission combined with the high sensitivity of transit light curves to small changes in the star-planet orbit make this measurement plausible. One should also note that the size of the apsidal precession signal increases dramatically with a longer baseline. In the far future, many planets will have measured apsidal precession rates (like eclipsing binary stars) and inferred $k_{2 p}$ values. Incorporating these measurements into interior models holds significant promise for greater understanding of all extra-solar planets.

\section{References}

Barnes, J. W. \& Fortney, J. J. 2003, ApJ, 588, 545

Beatty, T. G. \& Gaudi, B. S. 2008, arXiv:astro-ph/0804.1150

Bodenheimer, P., Lin, D. N. C., \& Mardling, R. A. 2001, ApJ, 548, 466

Borucki, W. et al. 2008, these Proceedings

Burrows, A., Hubeny, I., Budaj, J., \& Hubbard, W. B. 2007, ApJ, 661, 502

Claret, A. 1995, Ap\&SSS, 109, 441

Guillot, T., Santos, N. C., Pont, F., Iro, N., Melo, C., \& Ribas, I. 2006, Astron. Let., 453, L21, arXiv:astro-ph/0605751

Heyl, J. S. \& Gladman, B. J. 2007, MNRAS, 377, 1511

Jordan, A. \& Bakos, G. A. 2008, arXiv:astro-ph/0806.0630

Knutson, H. A. et al. 2007, Nature, 447, 183

Koch, D. et al. 2006, ApESSS, 304, 391

Kozai, Y. 1959, AJ, 64, 367

López-Morales, M. \& Seager, S. 2007, ApJ (Letters), 667, L191

Miralda-Escudé, J. 2002, ApJ, 564, 1019

Pál, A. \& Kocsis, B. 2008, arXiv:astro-ph/0806.0629

Ragozzine D. \& Wolf, A. S., in prep.

Sasselov, D. D. 2003, ApJ, 596, 1327

Sterne, T. E. 1939, MNRAS, 99, 451 